\title{
N-BODY SIMULATION OF LARGE SCALE STRUCTURE IN THE UNIVERSE WITH OSCILLATING SCALAR FIELD
}

\author{
K. KAWABATA, H. TAGUCHI AND Y. ANDOH \\ Department of Physics, Science University of Tokyo \\ 1-3 Kagurazaka, Shinjuku-ku, Tokyo 162, Japan
}

\begin{abstract}
Using the particle-mesh method with $128^{3}$ grids and $64^{3}$ particles, we have carried out a number of $\mathrm{N}$-body simulations of the large scale structure for the cosmological model proposed by Fukuyama et al.(1996): this model contains the matter $\left(\Omega_{0}\right)$ as well as a scalar field $(\phi)$ with a finite mass that couples non-minimally with the scalar curvature $R$ through the form of $\frac{1}{2} \eta \phi^{2} R$, where $\eta(=-80)$ is the coupling constant.

For simplicity, we have adopted the same values as those employed by Fukuyama et al.(1996) for all the parameters other than $\Omega_{0}$, fo which we have varied from 0.001 to 0.15 . In performing our simulation, we further assume that only the density of matter $\rho$ spatially fluctuates. The initial condition is created by perturbing the homogeneous distribution of the particles by means of the random Gaussian Harrison-Zeldovich spectrum.

The two-point correlation function $\xi(r)$ is then compputed for each of the resulting structures to compare with the observational data(Davis and Peebles, 1983). It is interesting to note that the structure formation appears to be achieved rather straightforwardly with the scalar field model. However, in order to yield an agreement between the theoretical and the observational two-point correlation functions, we seem to require the $\Omega_{0}$ value much larger than 0.01 adopted by Fukuyama et al.(1996).
\end{abstract}

\section{References}

Davis,M. and Peebles, P.J.E., 1983, Ap.J., 267, 465.

Fukuyama, T., Hatakeyama, M., Miyoshi, M., Morikawa, M., and Nakamichi, A., 1996, Astro-ph/9608097. Submitted to Int. J. of Phys., D. 